\section{AB016. A double-blind, placebo-controlled, phase 2 trial of a novel toll-like receptor 7/8/9 antagonist (IMO-8400) in dermatomyositis}

\author{
Yoo Jung Kim ${ }^{1}$, Elena Schiopu ${ }^{2}$, Katalin Dankó ${ }^{3}$, \\ Tahseen Mozaffar ${ }^{4}$, Srinivas Chunduru ${ }^{5}$, \\ Kirstin Lees ${ }^{5}$, Namita Goyal ${ }^{4}$, Jeffrey Sarazin ${ }^{2}$, \\ David F. Fiorentino ${ }^{1}$, Kavita Y. Sarin ${ }^{1}$
}

${ }^{1}$ Department of Dermatology, Stanford University School of Medicine, Redwood City, CA, USA; ${ }^{2}$ Department of Rheumatology, University of Michigan, Ann Arbor, MI, USA; ${ }^{3}$ Department of Clinical Immunology, Medical Faculty, University of Debrecen, Debrecen, Hungary; ${ }^{4}$ Department of Neurology, University of California Irvine, Irvine, CA, USA; ${ }^{5}$ Idera Pharmaceuticals, Inc., Exton, PA, USA

Correspondence to: Kavita Y. Sarin; David F. Fiorentino. Department of Dermatology, Stanford University School of Medicine, Redwood City, CA, USA. Email: ksarin@stanford.edu; fiorentino@stanford.edu.

Background: Dermatomyositis (DM) is an inflammatory disease of skin and muscle. Increased interferon (IFN) signaling is a prominent feature of DM, but the mechanisms leading to IFN production in DM are not understood. As toll-like receptor (TLR) 7/8/9 activation can lead to type I IFN production, TLR7/8/9 antagonism may provide therapeutic benefit in DM.

Methods: A double-blind, randomized, placebo-controlled, 24-week trial of IMO-8400 [a novel oligonucleotide TLR7/8/9 antagonist (Idera Pharmaceuticals, Inc.)] was conducted with 30 participants meeting definite or probable criteria of Bohan and Peter for DM. Participants were randomized to treatment with IMO-8400 $0.6 \mathrm{mg} / \mathrm{kg}$, IMO$84001.8 \mathrm{mg} / \mathrm{kg}$, or placebo. The primary endpoint was the change in the Cutaneous Dermatomyositis Disease Area and Severity Index (CDASI) activity score. Exploratory analysis included type I IFN signaling and the 5-D Itch Scale. Blood and skin samples were obtained at baseline and end of treatment to measure changes in type I IFN signaling.

Results: CDASI activity scores decreased in all arms by the end of the trial, per repeated measures mixed model analysis: -9.3 in $0.6 \mathrm{mg} / \mathrm{kg},-8.8$ in $1.8 \mathrm{mg} / \mathrm{kg}$, and -7.3 in placebo. We observed no change in skin and blood type I IFN signature scores or CDASI activity scores across treatment arms. We found an association between CDASI and skin IFN signature scores $(\beta=12.9, \mathrm{P}=0.0002)$, an association between 5-D Itch Scale and skin IFN signature scores (Rho $=0.65, \mathrm{P}<0.0001$ ), a lack of association between 5 -D Itch Scale and blood IFN signature scores $($ Rho $=0.22$, $\mathrm{P}=0.24)$, and a positive trend that did not reach significance between CDASI and 5-D Itch Scale scores. Five patients experienced treatment-emergent adverse effects prompting discontinuation: 3 in low-dose (abdominal discomfort/flu, anxiety, urticaria), 1 in high-dose (thrombocytopenia), and 1 in placebo (muscle weakness).

Conclusions: IMO-8400 did not significantly reduce DM disease activity or type I IFN expression. Our study demonstrates that cutaneous DM disease activity may be better studied through skin biopsies, rather than peripheral blood draws, and that type I IFN signaling could be a potential target in improving pruritus in $\mathrm{DM}$ patients.

Keywords: Clinical research; dermatomyositis (DM); toll-like receptors (TLRs)

doi: 10.21037/atm.2021.AB016

Open Access Statement: This is an Open Access article distributed in accordance with the Creative Commons Attribution-NonCommercial-NoDerivs 4.0 International License (CC BY-NC-ND 4.0), which permits the noncommercial replication and distribution of the article with the strict proviso that no changes or edits are made and the original work is properly cited (including links to both the formal publication through the relevant DOI and the license). See: https://creativecommons.org/licenses/by-nc-nd/4.0/.

Cite this abstract as: Kim YJ, Schiopu E, Dankó K, Mozaffar T, Chunduru S, Lees K, Goyal N, Sarazin J, Fiorentino DF, Sarin KY. A double-blind, placebo-controlled, phase 2 trial of a novel toll-like receptor 7/8/9 antagonist (IMO-8400) in dermatomyositis. Ann Transl Med 2021;9(5):AB016. doi: 10.21037/atm.2021.AB016 